\title{
Tumor burden monitoring using cell-free tumor DNA could be limited by tumor heterogeneity in advanced breast cancer and should be evaluated together with radiographic imaging
}

José Angel García-Saenz¹, Patricia Ayllón'1, Marion Laig², Daniel Acosta-Eyzaguirre³, Marta García-Esquinas, Myriam Montes ${ }^{4}$, Julián Sanz ${ }^{6}$, Miguel Barquín, Fernando Moreno ${ }^{1}$, Vanesa Garcia-Barberan ${ }^{1}$, Eduardo Díaz-Rubio ${ }^{1}$, Trinidad Caldes ${ }^{1 *}$ and Atocha Romero ${ }^{1,7^{*}}$ (I)

\begin{abstract}
Background: Accurate measurement of tumor burden in breast cancer disease is essential to improve the clinical management of patients. In this study, we evaluate whether the fluctuations in the fraction of PIK3CA mutant allele correlates with tumor response according to RECIST criteria and tumor markers quantification.

Methods: Eighty six plasma samples were analyzed by digital PCR using Rare Mutation Assays for E542K, E545K and H1047R. Mutant cfDNA and tumor markers CA15-3 and CEA were compared with radiographic imaging.

Results: The agreement between PIK3CA mutation status in FFPE samples and circulating tumor DNA (ctDNA) was moderate $(K=0.591 ; 95 \% \mathrm{IC}=0.371-0.811)$. Restricting the analysis to the metastatic patients, we found a good agreement between PIK3CA mutation status assessed in liquid and solid biopsy ( $K=0.798$ 95\%; IC $=0.586-1$ ). ctDNA showed serial changes with fluctuations correlating with tumor markers 15.3 and CEA in 7 out of 8 cases with Pearson correlation coefficients ranging from 0.99 to 0.46 and from 0.99 to 0.38 respectively. Similarly, fluctuations in the fraction of PIK3CA mutant allele always correlated with changes in lesion size seen on images, although in two cases it did not correlate with treatment responses as defined by RECIST criteria.

Conclusion: oncogenic mutation quantification in plasma samples can be useful to monitor treatment outcome. However, it might be limited by tumor heterogeneity in advanced disease and it should be evaluated together with radiographic imaging.
\end{abstract}

Keywords: Breast cancer, dPCR, PIK3CA, cfDNA

\section{Background}

Tumor biopsies have been and currently are the keystone for biomarker testing [1]. However, some limitations of tumor biopsies are still significant, such as difficulties in obtaining tissue samples, the invasiveness of the process or tumor heterogeneity, which might

\footnotetext{
* Correspondence: trinidad.caldes@salud.madrid.org; atocha10@hotmail.com Jose Angel Garcia-Saenz, Fernando Moreno, Vanesa Garcia-Barberan, Eduardo Diaz-Rubio and Trinidad Caldes are members of CIBERONC network.

'Medical Oncology Department, Hospital Clínico San Carlos, Madrid, Spain

Full list of author information is available at the end of the article
}

compromise the efficacy of targeted-therapies, based on a single biopsy $[2,3]$.

Tissue cells release circulating free DNA (cfDNA) into the bloodstream. Specifically, tumor-derived DNA (ctDNA) can be detected in plasma samples by identifying specific genetic alterations that tumors harbor, using highly sensitive technologies, such as digital PCR (dPCR), with the advantage that the aforementioned limitations can be overcome. In addition, quantification of tumor-specific mutations in liquid biopsies has been shown to correlate with tumor burden and there is growing evidence 
suggesting that ctDNA monitoring can predict treatment outcome [4-9].

At present, tumor response to treatment is commonly evaluated according to RECIST criteria. However, several studies have pointed out the limitations that radiology assessment may have when assessing tumor response, especially in estrogen receptor (ER) positive breast cancer patients [10-13].

The PIK3CA gene encodes the p110 $\alpha$ catalytic subunit of PI3K protein, and is mutated in over one-third of breast cancer cases [14]. Specifically, $40 \%$ of hormone dependent cancers harbor activating mutations in PIK3CA [14-17], making this gene an attractive candidate for tumor monitoring by tumor-specific mutation quantification in plasma samples. Mutations occur predominantly in the helical domain (generally E542K and E545K) and the kinase domain (generally H1047R) and account for $70 \%$ of all PIK3CA mutations in breast tumors [14-17]. Hotspot mutations have been shown to confer oncogenic features although the prognostic/predictive significance of PIK3CA mutations remains controversial.

In this study, we compare the clinical information provided by PIK3CA mutation quantification using arraybased $\mathrm{dPCR}$, in plasma samples from ER positive breast cancer patients, with tumor markers 15-3 and CEA and computed tomography (CT) scan assessments.

\section{Methods}

\section{Study population}

A total of 49 patients with estrogen receptor (ER) positive breast cancer were prospectively enrolled in the study after signing the appropriate informed consent. Patients agree to the publication of anonymized data. The study protocol was approved by the Hospital Clinico San Carlos Ethics Committee (internal code 13/383-E). Eligibility criteria comprised the following: women aged between 18 and 80 years with a pathologically confirmed diagnosis invasive breast carcinoma, clinical stage IIB-IIIB or IV and availability of the archived tissue for genotyping at the Hospital Clinico San Carlos Biobank. Blood samples were collected at the beginning of a treatment that the patient was going to initiate when included in the study. In 43 cases, the tumor specimen collected was the primary breast tumor; a biopsy of a FFPE metastatic breast cancer lesion was available in the remaining 6 patients. Information concerning demographics, clinicopathological features (stage, grade, estrogen and progesterone receptor status and HER2 status), courses of treatment(s), and vital status was obtained from the clinical and pathology reports (Additional file 1).

\section{Laboratory analysis}

Eighty six blood samples were collected in BD Vacutainer ${ }^{\circ} \mathrm{CPT}^{\mathrm{m}}$ Cell Preparation Tube with Sodium Heparin (containing a gel barrier). Hemolyzed samples were discarded for further analyses. Plasma was derived from blood samples by centrifugation within 3 hours after blood extraction. cfDNA was isolated from $3 \mathrm{ml}$ of plasma using the QIAamp Circulating Nucleic Acid Kit (Qiagen, Valencia, CA), according to manufacturer's instructions. cfDNA was eluted in $35 \mu \mathrm{L}$ of the supplied elution buffer. The eluate was reloaded in the column and eluted by maximum speed centrifugation in order to increase the amount of cfDNA. Samples were then analyzed by digital PCR, using Rare Mutation Assays ${ }^{1}$ for E542K (AHD2BSD), E545K (AHABHHX) and H1047R (AHPAVCD) on QuantStudio ${ }^{\circ}$ 3D Digital PCR System (Applied Biosystems, South San Francisco, CA). For the $\mathrm{dPCR}$ reaction, $8 \mathrm{ul}$ of cfDNA was mixed with $0.5 \mathrm{ul}$ of the aforementioned 40X TaqMan assays and $10 \mathrm{ul}$ of $2 \mathrm{x}$ QuantStudio 3D Master Mix, in a 20ul reaction volume. Subsequently, 15 ul were loaded into QuantStudio 3D Digital PCR $20 \mathrm{~K}$ chips. The cycling conditions were as follows: initial denaturation at $96{ }^{\circ} \mathrm{C}$ for $10 \mathrm{~min}$, followed by 40 cycles at $56{ }^{\circ} \mathrm{C}$ for $2 \mathrm{~min}$, and $98{ }^{\circ} \mathrm{C}$ for 30 s, a step of $72{ }^{\circ} \mathrm{C}$ for $10 \mathrm{~min}$ and finally samples were kept at $22^{\circ} \mathrm{C}$ for at least $30 \mathrm{~min}$. Chip fluorescence was read twice. Results were analyzed with QuantStudio ${ }^{\circ}$ 3D Analysis Suite ${ }^{\text {mit }}$ Cloud Software. The automatic call assignments for each data cluster where manually adjusted when needed. The analysis of $\mathrm{dPCR}$ data was performed blinded to the clinical and tumor information by two independent investigators (AR and ML). Double positive data points (green data points) where only considered when FAM data points where visualized. The result of the assay is reported as mutant allele frequency (MAF) which is defined as the ratio of mutant DNA molecules vs the sum of wild-type (wt) DNA molecules and mutant DNA molecules. Samples were considered as positive when MAF was greater than $0.15 \%$. A wt control DNA was included in every run. All assays were validated using plasmid DNAs harboring the aforementioned the PIK3CA mutations (GeneArt, Thermo Fisher Scientific). Germline wt DNA from healthy donors was mixed with different mutant allele concentrations (i.e. $1 \%$, $0.5 \%, 0.1 \%, 0.05 \%)$ in order to estimate the Limit of quantitation (LOQ) and limit of detection (LOD). LOD and LOQ were calculated based on the standard deviation of the response and the slope according to ICH Q2(R1) guideline (http://www.ich.org/products/guidelines/quality/article/ quality-guidelines.html). The standard deviation of the response was calculated based on standard error of the $y$ intercept. Additionally, LOD and LOQ were estimated based on blank measurements. In this case, LOD was expressed as the average of MAF corresponding to the wt samples plus three standard deviation and LOQ was the average of MAF corresponding to the wt samples plus ten standard deviations. Two replicates were performed for all patients. 
For the analyses of FFPE tumor and metastasis samples, a hemotoxylin and eosin-stained slide was reviewed by a pathologist to confirm the presence of tumor cells within the section. Overall, the percentage of tumor cells in the analyzed samples ranged from 80 to $90 \%$. Subsequently, DNA was purified from four 5-mm-thick unstained FFPE sections according to the QIAamp DNA FFPE Tissue Kit (Qiagen, Valencia, CA) protocol.

The identification of PIK3CA mutations on FFPE samples was performed using COBAS $^{\circ}$ PIK3CA Mutation Test (Roche Molecular Systems, Branchburg, NJ). The test is intended to detect R88Q in exon $1, \mathrm{~N} 345 \mathrm{~K}$ in exon 4, C420R in exon 7, E542K, E545X (E545A, E545D,E545G, and E545K), Q546X (Q546E, Q546K, Q546L, and Q546R) in exon 9, and M1043I, H1047X (H1047L, H1047R, and H1047Y), and G1049R in exon 20 when the percent mutation is equal or greater than $5 \%$. PIK3CA mutation status was further confirmed by the aforementioned TaqMan assays on the QuantStudio ${ }^{\circ}$ 3D Digital PCR System. Conflicting samples were further amplified and sequenced. Primers and conditions used are available upon request. PCR products were then sequenced on the ABI 3130 genetic analyzer (Applied Biosystems, Foster City, CA), using the BigDye Terminator v1.1 Cycle Sequencing Reaction kit (Applied Biosystems, Foster City, CA) following the manufacturer's protocol kit.

Serum samples were used to measure the levels of CA 15-3 antigen and CEA (Carcinoembryonic Antigen) using the high throughput immunoassay analyzer UniCelDxI 800 (Beckman Coulter), according to manufacturer's instructions. In brief, the assay is a two-step sandwich immunoassay using direct, chemiluminescent technology.

\section{Response evaluation}

Mammogram, ultrasound-based, magnetic resonance imaging (MRI), computed tomography (CT) and Positron emission tomography (PET)-CT tumor measurements were obtained, as clinically indicated. The clinical response was evaluated according to RECIST v1.1 criteria comparing radiological assessments. Complete response (CR) was defined as resolution of all palpable, visible or radiology abnormalities in the breast and regional lymph nodes. Partial response (PR) was defined as a decrease of $\geq 30 \%$ in the sum of the longest diameter in the breast and regional lymph nodes. Stable disease (SD) was assigned to patients who did not meet the criteria for CR, PR or PD. Clinical Progressive disease (PD) was defined as an increase of at least $20 \%$ in the sum of the longest diameter in the breast and regional lymph nodes or progression of other clinical manifestations of disease.

\section{Statistic analysis}

Continuous variables are presented as means \pm standard deviations (SD) and discrete variables as proportions.
The weighted quadratic kappa coefficient values and the corresponding 95\% confidence intervals (95\% CI) were estimated in order to measure the strength of agreement between methodologies. The strength of agreement is judge to be minor when the $\mathrm{K}$ values are between 0.00 and 0.20; fair, 0.21 and 0.40 ; moderate, 0.41 and 0.60 ; good, 0.61 and 0.80 and nearly perfect, 0.81 and 1.00 . Correlation between quantitative variables was evaluated using the Pearson correlation coefficient. The correlation among the quantitative variables was evaluated with simple linear regression analysis. $P<0.05$ was considered to be statistical significance. The statistical analysis was performed using software R 3.0.1.

\section{Results}

Assay performance and agreement between methodologies

We report data from 49 patients with ER positive breast cancer undergoing therapy that were prospectively enrolled in the study. The pathological characteristics of study population are summarized in Table 1 and Additional file 1. The source of archival tissue sample was the primary tumor for $43(87.8 \%)$ patients and biopsy of a distant metastasis for 6 (12.2\%). DNA

Table 1 Clinicopathological features of the study population

\begin{tabular}{ll}
\hline Clinicopathologic Characteristics & \\
\hline Age at diagnosis (years) & $62(30-80)$ \\
Median (range) & \\
Histology (n (\%)) & $44(90 \%)$ \\
Ductal & $5(10 \%)$ \\
Lobular & \\
Histologycal grade (n (\%)) & $39(80 \%)$ \\
I and II & $10(20 \%)$ \\
III & \\
UICC stage (n (\%)) & $6(12 \%)$ \\
IIB & $11(22 \%)$ \\
IIIA + IIIB & $32(65 \%)$ \\
IV & \\
ER status (n (\%)) & $49(100 \%)$ \\
positive & $0(0 \%)$ \\
negative & \\
PR status(n (\%)) & \\
positive & $33(67 \%)$ \\
negative & $16(33 \%)$ \\
HER2 status (n(\%)) & \\
positive & \\
negative & $41(84 \%)$ \\
\hline Abbreviations: ER estrogen receptor PR progesterone receptor $H E R 2 ~ e r b-b 2$
\end{tabular}

WUTions: ER estrogen receptor, $P R$ progesterone receptor, HER2 erb-b2 receptor tyrosine kinase 2, UICC Union for International Cancer Control 
isolated from archival-tumor tissue samples was analyzed to identify PIK3CA somatic mutations. According to COBAS, among the analyzed tumors, 11 (22.4\%) harbored the mutation H1047R, 5 (10.2\%) harbored the E545K and 4 (8.1\%) harbored the E542K. These are expected frequencies according to previously published data. Interestingly, we did not encounter any other less frequent PIK3CA mutation that could be detected by COBAS methodology. Subsequently, the three PIK3CA hot spot mutations were screened in FFPE derived samples using QuantStudio 3D system. The proportion of observed agreement between COBAS and QuantStudio3D system when assessing the PIK3CA mutation status in the archival tumor was $100 \%$ $(\mathrm{K}=1)$ (Additional file 1).

Next, we evaluated the detection sensitivity of TaqMan assays. For sensitivity assays plasmid carrying E542K, E545K and H1047R mutations were mixed at different allele concentrations with wt DNA extracted from peripheral blood cells form healthy donors. Mutant allele frequencies correlated with the expected mutant allele frequencies in E542K assay, E545K assay and H1047R assay (Pearson's correlation coefficient, 0.9985, 0.9966 and 0.9991 respectively). LOD for E542K, E545K and H1047R assays were $0.04,0.05$ and $0.03 \%$ respectively. LOQ for E542K, E545K and H1047R assays were 0.13, 0.15 and $0.08 \%$ respectively (Additional file 2). Additionally, wt DNA from healthy donors were used to evaluate the false positive signals. The mean of mutant allele fraction of wt DNA form healthy individuals was $0.01 \%$ for E542K assay, $0.00 \%$ for E545K assay and $0.01 \%$ for H1047R.

Finally, we evaluated the agreement between PIK3CA mutation status of FFPE samples and the matched plasma sample. The median time between blood collection at study entry and time that the tumor biopsy was obtained from the patient was 7 years (range $=0-15$ years). Importantly, cfDNA from all plasma samples was successfully amplified. Representative plots for the three assays, performed on cfDNA, are displayed in Additional file 3. The ratio of mutant DNA molecules vs total DNA molecules ranged from 0.19 to $53.4 \%$ in the positive sample cases. The median of PIK3CA mutated copy number in positive samples was 1496 copies/ml (range: 63.3-92742).

Overall, we found a moderate agreement between PIK3CA mutation status in plasma and FFPE tumor samples $(K=0.591 ; 95 \%$ IC $=0.371-0.811)$. Specifically, we found that in 9 samples, the assay failed to detect on cfDNA the mutation identified in the tumor sample (data obtained from Additional file 1). However, we did not find any mutation in the plasma that was not present in the FFPE sample (sensitivity 55\%, specificity $100 \%$, positive predictive value $100 \%$, and negative predictive value $76 \%$ ). Restricting the analysis to the metastatic patients, we found a good agreement between PIK3CA mutation status assessed in liquid and solid biopsy $\quad(\mathrm{K}=0.798 \quad 95 \% ; \quad \mathrm{IC}=0.586-1) \quad$ (data obtained Additional file 1). In this case, the cfDNA assay failed to detect the tumor mutation in 3 samples (sensitivity $77 \%$, specificity $100 \%$, positive predictive value $100 \%$ and negative predictive value $86 \%$ ).

\section{Correlation with tumor markers}

In 8 breast cancer patients with stage IV disease in which PIK3CA somatic mutation was identified in both FFPE and plasma samples, mutant cfDNA and tumor markers CA15-3 and CEA were quantified in a total of 27 serial plasma samples (Table 2). Circulating tumor DNA (ctDNA) was identified in 21 of the 27 plasma samples analyzed. Tumor markers CA15-3 and CEA levels were evaluated at all-time points in all 8 patients. Circulating tumor DNA showed serial changes with fluctuations correlating with tumor markers in 7 cases. In one case (from now on patient 3) changes in PIK3CA mutation quantification were in the opposite direction than tumor markers. In patients in which tumor markers CA15-3 and CEA and PIK3CA mutant DNA fluctuated in the same way, Pearson correlation coefficients ranged from 0.99 to 0.46 and from 0.99 to 0.38 respectively (mean 0.82 for CA $15-3$ and 0.79 for CEA).

\section{Concordance between imaging assessments and PIK3CA mutation quantification in plasma}

As an exploratory analysis, in the aforementioned 8 patients we compared the performance of PIK3CA assays with measurable disease (as defined by RECIST criteria). The median follow-up was 7.5 months. Overall, changes in ctDNA were in concordance with treatment responses observed in imaging. Partial responses were recognized in two patients (patient 25 and patient 44) during the follow-up period. In both cases, a reduction of mutant allele fraction in plasma samples was evidenced (Additional file 4: Figure S1 and Fig. 1b respectively). Similarly, progressive disease (PD) was documented in 4 women (patient 44, patient 2, patient 31, patient 3) during the study. An Increase in PIK3CA mutant allele fraction reflected progressive disease in 2 patients (patient 2 represented in Fig. 1a and patient 44 represented in Fig. 1b). Noteworthy, in one case (patient 31), although H1047R mutation quantification went down to $0 \%$ over the course of treatment (from 0.19 to $0 \%$; Table 2) correlating with tumor markers CA15.3 and CEA variations and with the decrease in size of the pulmonary metastases and the axillary lymphadenopathy, the contrast-enhanced CT scan from the same period of time demonstrated that the lytic bone metastases increased in size (Fig. 1c). Similarly, in case of Patient 3, the hiliar and mediastinal adenopathies decreased in size from 13 to $8 \mathrm{~mm}$ to 7 and $4 \mathrm{~mm}$ 
Table 2 ctDNA and tumor markers measurements in 8 breast cancer patients with stage IV disease

\begin{tabular}{|c|c|c|c|c|c|c|c|}
\hline Patient & Tracked mutation & Sample & Target/Total & mutated copies/mL & CA $15.3(\mathrm{U} / \mathrm{ml})$ & CEA (ng/ml) & Figure \\
\hline \multirow[t]{3}{*}{25} & \multirow[t]{3}{*}{ H1047R } & March 072014 & $18.36 \%$ & 54398 & 97 & 27 & \multirow[t]{3}{*}{ Additional file 4: Figure S1 } \\
\hline & & Oct 132014 & $0.11 \%$ & 12.1 & 32.8 & 7.1 & \\
\hline & & Nov 032014 & $0 \%$ & 0 & 32.3 & 7.5 & \\
\hline \multirow[t]{4}{*}{44} & \multirow[t]{4}{*}{ H1047R } & Aug 292014 & $1.78 \%$ & 310.5 & 126 & 1.6 & \multirow[t]{4}{*}{ Fig. 1b } \\
\hline & & Sep 172014 & $1.85 \%$ & 975 & 997.2 & 5.2 & \\
\hline & & Oct 232014 & $2.08 \%$ & 1203.2 & 1219 & 11.6 & \\
\hline & & Nov 192014 & $0.81 \%$ & 102.9 & 487.3 & 4.8 & \\
\hline \multirow[t]{3}{*}{15} & \multirow[t]{3}{*}{ H1047R } & Feb 182014 & $0.36 \%$ & 24.7 & 68.9 & 4.7 & \multirow[t]{3}{*}{ Additional file 4: Figure S3 } \\
\hline & & May 292014 & $0.32 \%$ & 30.6 & 58.6 & 4.3 & \\
\hline & & Aug 272014 & $0.03 \%$ & 25.6 & 39.4 & 3.3 & \\
\hline \multirow[t]{4}{*}{2} & \multirow[t]{4}{*}{ H1047R } & Dec 032013 & $2.39 \%$ & 149.6 & 96.8 & 5.7 & \multirow[t]{4}{*}{ Fig. 1a } \\
\hline & & Jan 022014 & $3.93 \%$ & 77.3 & 91.6 & 12.7 & \\
\hline & & Jan 302014 & $4.39 \%$ & 3082.1 & 100.6 & 13.9 & \\
\hline & & March 032014 & $9.61 \%$ & 3629.3 & 138.1 & 19.8 & \\
\hline \multirow[t]{4}{*}{31} & \multirow[t]{4}{*}{ H1047R } & March 272014 & $0.19 \%$ & 13.5 & 89.3 & 22.1 & \multirow[t]{4}{*}{ Fig. 1c } \\
\hline & & Aug 292014 & $0 \%$ & 0 & 30.7 & 6.2 & \\
\hline & & Oct 022014 & $0 \%$ & 0 & 28.8 & 5.1 & \\
\hline & & Nov 032014 & $0 \%$ & 0 & 17.5 & 4.4 & \\
\hline \multirow[t]{2}{*}{11} & \multirow[t]{2}{*}{ E545K } & March 062014 & $27.19 \%$ & 9274.2 & 86 & 108 & \multirow[t]{2}{*}{ Additional file 4: Figure S2 } \\
\hline & & April 092014 & $6.61 \%$ & 235.9 & 66 & 86 & \\
\hline \multirow[t]{4}{*}{3} & \multirow[t]{4}{*}{ E545K } & Dec 022013 & $4.23 \%$ & 412.5 & 57.6 & 2.8 & \multirow[t]{4}{*}{ Fig. 2} \\
\hline & & March 122014 & $3.07 \%$ & 1839.5 & 60.8 & 3 & \\
\hline & & Sep 102014 & $1.97 \%$ & 165.1 & 114.6 & 15.1 & \\
\hline & & Dec 152014 & $0.76 \%$ & 143.5 & 179.5 & 9 & \\
\hline \multirow[t]{3}{*}{6} & \multirow[t]{3}{*}{ E542K } & Feb 102014 & $0.24 \%$ & 63.30 & 115.6 & 3.9 & \multirow[t]{3}{*}{ Additional file 4: Figure S4 } \\
\hline & & Apr 042014 & $0 \%$ & 0 & 88.5 & 2.9 & \\
\hline & & Sep 052014 & $0 \%$ & 0 & 45 & 1.7 & \\
\hline
\end{tabular}

respectively, correlating with E545K mutation quantification that significantly decreased from $3.07 \%$ (CI: $2.71 \%-3.465 \%$ ) to $1.97 \%$ (CI: $1.343 \%-2.88 \%$ ) (Table 2 ) in the corresponding plasma samples. However, the pulmonary metastases increased in number and size during the same period of time correlating with tumor markers quantification (Fig. 2). Finally, stable disease was documented in two patients (patient 15 and patient 6) in which tumor circulating free DNA fluctuated in the same way that tumor markers fluctuated (Additional file 4: Figure S3 and Figure S4).

\section{Discusion}

In this study, we have tested the feasibility of arraybased digital PCR to detect and quantify tumor specific mutations in plasma samples and we have documented the correlation between PIK3CA mutation quantification and tumor responses assessed by RECIST criteria.
Several groups $[6,18-21]$ have reported the utility of cfDNA as a source for tumor mutation detection. As described by others, we found a good agreement ( $\mathrm{K}=$ 0.798) between plasma and FFPE samples when assessing PIK3CA mutation status in advanced patients, using array-based dPCR. However, in our experience the agreement was lower when early stages patients were included. Consistent with this, Oshiro et al have recently reported that PIK3CA mutations were detected in only $22.7 \%$ of serum samples from early-stage breast cancer patients with tumors harboring a PIK3CA mutation using array-based dPCR. Of important note, in that study, the authors proved that the sensitivity of the assay was $0.01 \%$ [22]. In the same way, Bettegowda $C$ et al have reported ctDNA was detectable in $>75 \%$ patients with advanced breast cancer disease whereas the frequency of cases with detectable ctDNA was $50 \%$ in early stages [23]. On the contrary, other researchers have reported a high agreement between liquid and solid biopsy 

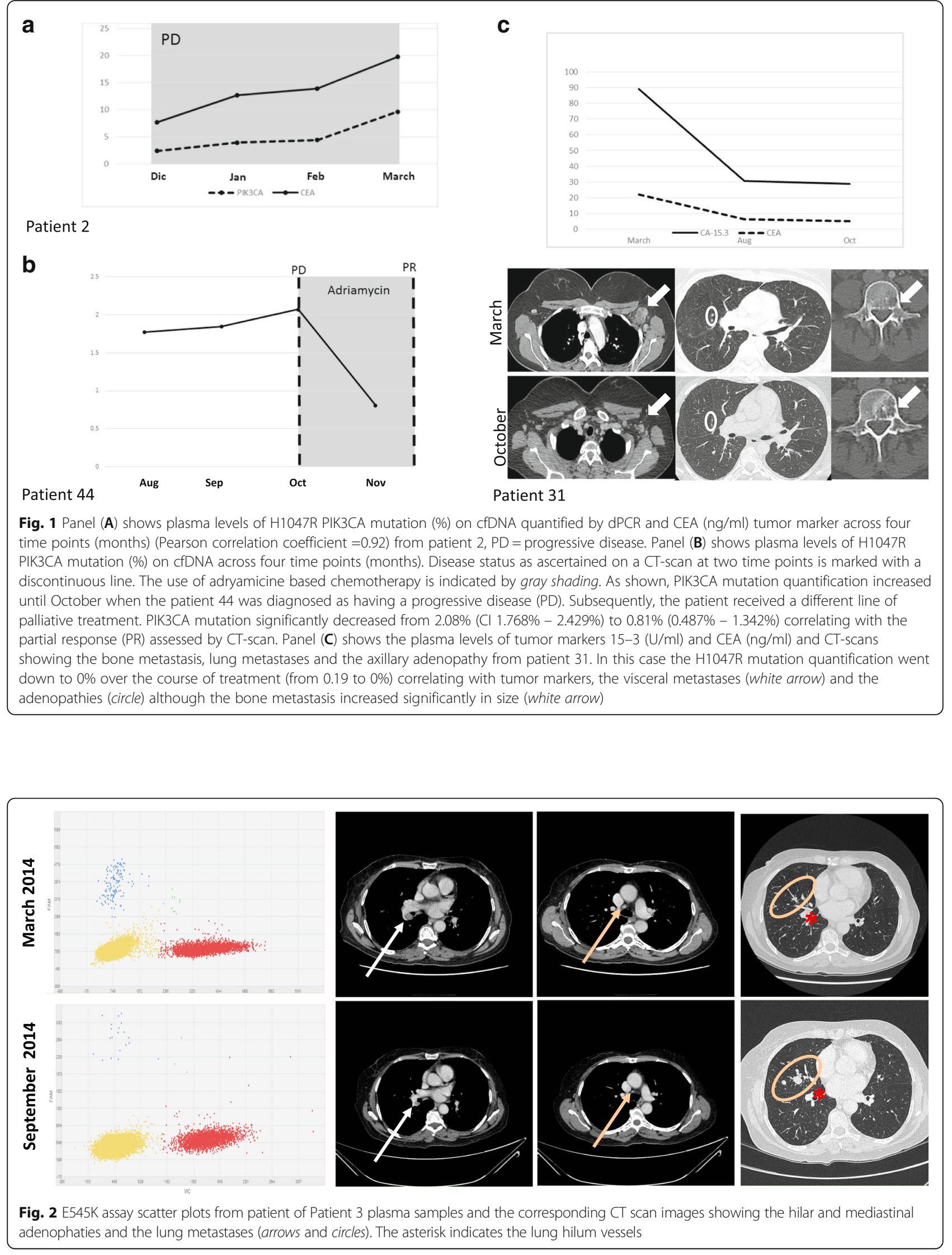
for PIK3CA mutation status assessment in early stages using droplet digital PCR [19]. Nevertheless, the sample size of our study was limited and larger sized cohorts are needed to clarify this issue. To our knowledge, there is no data comparing the aforementioned methodologies (droplet vs array). Such studies would be of particular interest in order to determine the best strategy for biomarker testing to guide targeted cancer therapies using liquid biopsy.

The evaluation of tumor response to treatment that identifies patients early on that do not benefit from therapies remains a public health challenge. The capacity of serial monitoring of ctDNA to track tumor burden has been previously addressed by several researchers $[6,13,24]$. In this way, Dawson SJ et al analyzed PIK3CA mutation quantification in 13 cases [6]. According to the authors, PIK3CA mutation quantification correlated with changes in tumor burden. Similarly, we found that in 6 out of 8 cases the level of PIK3CA mutations correlated with treatment responses according to RECIST criteria. In the two remaining cases, the discordance could be reflective of tumor evolution. Indeed, the radiology evaluation of the disease revealed a different sensitivity of the metastatic lesions to treatment, highlighting the issue of heterogeneity within advance disease. Similarly, Higgins [18] et al reported a $27.5 \%$ discordance among 51 patients with recurrent metastatic disease prospectively tested by BEAMing in blood compared with standard sequencing of archival tissue. Yet, our findings were consistent with responses seen on imaging as we could impute the lesions that most probably harbored the PIK3CA mutation. It is well known that breast cancer is a heterogeneous disease and that molecular profile of cancer can change over time $[25,26]$. This fact might limit usefulness of ctDNA to monitor response to treatment, especially when the mutation tracked is not targeted by therapy.

In any case, our results suggest that changes in mutant allele fraction in liquid biopsy should be analyzed together with imaging data in order to make mutation analysis informative.

\section{Conclusions}

In this study, we have shown that fluctuations in the fraction of PIK3CA mutant allele always correlated with changes in lesion size seen on images, although not always with treatment responses, as defined by RECIST criteria. Our findings suggest that information provided by oncogenic mutation quantification in plasma samples could be limited by tumor heterogeneity in advanced disease and should be evaluated together with radiographic imaging.

\section{Endnotes}

${ }^{1}$ For Research Use Only. Not for use in diagnostic procedures.

\section{Additional files}

Additional file 1: Clinical and pathological variables. (XLSX $16 \mathrm{~kb})$

Additional file 2: Limit of detection and limit of quantification estimation according to ICH guidelines. (XLSX $30 \mathrm{~kb}$ )

Additional file 3: Performance of PIK3CA assays on cfDNA. Data from sample chips are displayed in a scatter plot based on color of FAM and VIC events. Plots $A, C$ and $E$ correspond to a negative samples. Plots B, D and F correspond to positive samples for the H1047R, E545K and E542K mutation respectively. The mutation is labeled with FAM (blue data points) whereas wild-type is labeled with VIC (red data points). Yellow cluster represent the no amplification cluster. (PPTX $153 \mathrm{~kb}$ )

Additional file 4: Serial plasma PIK3CA mutation levels and treatment outcome, assessed according to RECIST criteria v.1.1. SD = stable disease, $\mathrm{PR}=$ partial response, $\mathrm{PD}=$ progressive disease of individual cases. (PPTX $78 \mathrm{~kb})$

\section{Abbreviations}

cfDNA: Circulating free DNA; dPCR: Digital polymerase chain reaction; MAF: Mutant allele fraction; FFPE Formalin-Fixed: Paraffin-embedded; ER: estrogen receptor

\section{Acknowledgements}

Not applicable.

\section{Funding}

This study was supported by grants RTICC12/0036/0041 and RTICC12/0036/ 0006, Instituto de Salud Carlos III, Spanish Ministry of Science and Innovation, and Fondo Europeo de Desarrollo Regional. AR is supported by Joan Rodés fellowship (JR14/00017) I + D + I 2013-2016 Instituto de Salud Carlos III. Spanish Ministry of Science and Innovation, and Fondo Europeo de Desarrollo Regional. We would like thank Enrique Camacho Garcia for his financial support. Any of funding bodies have not participated in the design of the study, data collection, analysis or interpretation of the data.

\section{Availability of data and materials}

The datasets used and/or analysed during the current study available from the corresponding author on reasonable request.

\section{Authors' contributions}

AR and JAGS conceived and coordinated the study. AR, ML, MGE, MM, JS, $J A G S, F M$ and EDR contributed to experimental design and data analysis. AR, PA, MB, DA, JS, FM and VGB carried out experiments. AR, TC and JAGS performed the statistical analyses. AR and TC contributed to manuscript preparation. All authors read and approved the final manuscript.

\section{Authors' information}

JAGS: medical oncologist, PA: PhD student (biologist), ML: product applications scientist, DAE: medical oncologist, MGE: radiologist, MM: radiologist, JS: pathologist, MB: PhD student (biologist), FM medical oncologist, VGB molecular biologist, EDR medical oncologist, TC laboratory medicine specialist, AR laboratory medicine specialist.

\section{Competing interests}

All authors have read the journal's authorship agreement and policy on disclosure of potential conflicts of interest. Marion Laig is employed by Thermo Fisher Scientific. Atocha Romero has received financial support for attending symposia from Thermo Fisher Scientific. The rest of the authors have declared no conflict of interest.

Consent for publication

Not applicable.

\section{Ethics approval and consent to participate}

The study was approved by the Hospital Clinico San Carlos Ethics Committee (internal code 13/383-E). Written informed consent to participate in the study was obtained from all patients. Experiments performed here comply with the current laws of Spain. 


\section{Publisher's Note}

Springer Nature remains neutral with regard to jurisdictional claims in published maps and institutional affiliations.

\section{Author details}

'Medical Oncology Department, Hospital Clínico San Carlos, Madrid, Spain. ${ }^{2}$ Thermo Fisher Scientific, Waltham, Massachusetts, USA. ${ }^{3}$ Medical Oncology Department, Centro Integral Oncológico Clara Campal HM hospitals, Madrid, Spain. ${ }^{4}$ Radiology Department, Hospital Clínico San Carlos, Madrid, Spain. ${ }^{5}$ Nuclear Medicine Department, Hospital Clínico San Carlos, Madrid, Spain. ${ }^{6}$ Pathology Department, Hospital Clínico San Carlos, Madrid, Spain. ${ }^{7}$ Medical Oncology Department, Hospital Universitario Puerta de Hierro Madrid, 28222 Majadahonda, Madrid, Spain.

Received: 31 August 2016 Accepted: 9 March 2017 Published online: 22 March 2017

\section{References}

1. Diamantis A, Magiorkinis E, Koutselini H. Fine-needle aspiration (FNA) biopsy: historical aspects. Folia Histochem Cytobio. 2003;47:191-7.

2. Gerlinger M, Rowan AJ, Horswell S, et al. Intratumor heterogeneity and branched evolution revealed by multiregion sequencing. N Engl J Med. 2012;366:883-92

3. Romero A, Acosta-Eyzaguirre A, Sanz J, et al. Identification of E545k mutation in plasma from a PIK3CA wild-type metastatic breast cancer patient by array-based digital PCR. cfDNA a powerful tool for biomarker testing in advance disease. Transl Res. 2015;166:783-7.

4. Diehl F, Schmidt K, Choti MA, et al. Circulating mutant DNA to assess tumor dynamics. Nat Med. 2008;14:985-90.

5. Wang JY, Hsieh JS, Chang MY, et al. Molecular detection of APC, K-ras, and p53 mutations in the serum of colorectal cancer patients as circulating biomarkers. World J Surg. 2004;28:721-6.

6. Dawson SJ, Tsui DW, Murtaza M, et al. Analysis of circulating tumor DNA to monitor metastatic breast cancer. N Engl J Med. 2013;368:1199-209.

7. Diaz Jr LA, Williams RT, Wu J, et al. The molecular evolution of acquired resistance to targeted EGFR blockade in colorectal cancers. Nature. 2012:486:537-40.

8. Sorensen BS, Wu L, Wei W, et al. Monitoring of epidermal growth factor receptor tyrosine kinase inhibitor-sensitizing and resistance mutations in the plasma DNA of patients with advanced non-small cell lung cancer during treatment with erlotinib. Cancer. 2014;120:3896-901.

9. Garcia-Murillas I, Schiavon G, Weigelt B, Ng C, Hrebien S, Cutts RJ, Cheang M, Osin P, Nerurkar A, Kozarewa I, Garrido JA, Dowsett M, Reis-Filho JS, Smith IE, Turner NC. Mutation tracking in circulating tumor DNA predicts relapse in early breast cancer. Sci Transl Med. 2015;7:302ra133.

10. Romero A, García-Sáenz JA, Fuentes-Ferrer M, et al. Correlation between response to neoadjuvant chemotherapy and survival in locally advanced breast cancer patients. Ann Oncol. 2013;24:655-61.

11. Ko ES, Han BK, Kim RB, et al. Analysis of factors that influence the accuracy of magnetic resonance imaging for predicting response after neoadjuvant chemotherapy in locally advanced breast cancer. Ann Surg Oncol. 2013; 20:2562-8.

12. Suzuki $\mathrm{C}$, Jacobsson $\mathrm{H}$, Hatschek $\mathrm{T}$, et al. Radiologic measurements of tumor response to treatment: practical approaches and limitations. Radiographics. 2008;28:329-44.

13. Charehbili A, Wasser MN, Smit VT, et al. Accuracy of MRI for treatment response assessment after taxane- and anthracycline-based neoadjuvant chemotherapy in HER2-negative breast cancer. Eur J Surg Oncol. 2014; 40:1216-21.

14. Stemke-Hale K, Gonzalez-Angulo AM, Lluch A, et al. An integrative genomic and proteomic analysis of PIK3CA, PTEN, and AKT mutations in breast cancer. Cancer Res. 2008;68:6084-91.

15. Banerji S, Cibulskis K, Rangel-Escareno C, et al. Sequence analysis of mutations and translocations across breast cancer subtypes. Nature. 2012;486:405-9.

16. Cancer Genome Atlas Network. Comprehensive molecular portraits of human breast tumour. Nature. 2012:490:61-70.

17. Campbell IG, Russell SE, Choong DY, et al. Mutation of the PIK3CA gene in ovarian and breast cancer. Cancer Res. 2004;64:7678-81.

18. Higgins MJ, Jelovac D, Barnathan $E$, et al. Detection of tumor PIK3CA status in metastatic breast cancer using peripheral blood. Clin Cancer Res. 2012;18:3462-9.
19. Beaver JA, Jelovac D, Balukrishna S, et al. Detection of cancer DNA in plasma of patients with early-stage breast cancer. Clin Cancer Res. 2014:20:2643-50.

20. Rothé F, Laes JF, Lambrechts D, et al. Plasma circulating tumor DNA as an alternative to metastatic biopsies for mutational analysis in breast cancer. Ann Oncol. 2014;25:1959-65.

21. Board RE, Wardley AM, Dixon JM, et al. Detection of PIK3CA mutations in circulating free DNA in patients with breast cancer. Breast Cancer Res Treat. 2010;120:461-7.

22. Oshiro C, Kagara N, Naoi Y, et al. PIK3CA mutations in serum DNA are predictive of recurrence in primary breast cancer patients. Breast Cancer Res Treat. 2015:150:299-307.

23. Bettegowda C, Sausen M, Leary RJ, et al. Detection of circulating tumor DNA in early- and late-stage human malignancies. Sci Transl. 2014;6:224ra24

24. Olsson E, Winter C, George A, et al. Serial monitoring of circulating tumor DNA in patients with primary breast cancer for detection of occult metastatic disease. EMBO Mol Med. 2015;7:1034-47.

25. Perou CM, Sørlie T, Eisen MB, et al. Molecular portraits of human breast tumours. Nature. 2000:406:747-52.

26. Romero A, Prat A, García-Sáenz JA, et al. Assignment of tumor subtype by genomic testing and pathologic-based approximations: implications on patient's management and therapy selection. Clin Transl Oncol. 2014;16:386-94.

\section{Submit your next manuscript to BioMed Central and we will help you at every step:}

- We accept pre-submission inquiries

- Our selector tool helps you to find the most relevant journal

- We provide round the clock customer support

- Convenient online submission

- Thorough peer review

- Inclusion in PubMed and all major indexing services

- Maximum visibility for your research

Submit your manuscript at www.biomedcentral.com/submit
Biomed Central 